\title{
Variational perturbation theory for electrolyte solutions
}

Leo Lue

\section{Introduction}

In typical treatments of the statistical mechanics of electrolyte solutions, the focus is on the particles (ions) in the system. The state of the system is labeled by the particle positions and orientations, and the partition function of the system is given by an integral over all these degrees of freedom. Another approach, which we pursue and present here, is to focus on the interaction potential generated by the particles, rather than on the particles themselves. We illustrate this approach by examining systems of particles interacting through electrostatic forces; however, these techniques can be used for a broad range of interaction potentials.

The remainder of the work is organized as follows. First we describe the representation of the partition function of an electrolyte system in terms of a functional integral over shapes of an interaction potential, rather than the positions and orientations of the ions in the system. As part of this, we will review electrostatics, and in particular, we will discuss the Green's function, which describes the propagation of the electric potential from a charge and plays a central role in the theory. The use and limitations of the mean field approximation to evaluate the functional integral are discussed. In the following section, we present the variational perturbation approximation, which is a method that overcomes some of the limitations of the mean-field approximation. Some general aspects of this approach are discussed. The resulting theory is then illustrated in Sec. 4 by applying it to the point charge model. The first order variational perturbation theory is compared to the Poisson-Boltzmann theory, and various features of the model, which are not captured within the classic Poisson-Boltzmann theory, are discussed, including the influence of image-charge or polarization interactions due to dielectric interfaces. Finally, the main points of

Leo Lue

Department of Chemical and Process Engineering, University of Strathclyde, James Weir Building, 75 Montrose Street, Glasgow G1 1XJ, United Kingdom e-mail: leo.lue@ strath.ac.uk 
this work is are summarized in Sec. 5, along with other applications and extensions of the theory.

\section{Development of the field theory}

We consider a multicomponent mixture of charged particles at fixed temperature $T$, volume $V$, and species chemical potentials $\mu_{\alpha}$. Associated with each particle of type $\alpha$ is a rigid charge density, given by $Q(\mathbf{r}, \boldsymbol{\Omega})$, where $\boldsymbol{\Omega}$ represents its orientation. These particles are embedded in a medium with a spatially varying dielectric constant $\varepsilon(\mathbf{r})$. In addition, there could be fixed charge distribution $\Sigma(\mathbf{r})$ through the system, for example, due to a charged boundary.

\subsection{Electrostatics}

The total charge density $\mathscr{Q}(\mathbf{r})$ in the system is composed of a contribution from the mobile particles and a contribution from a fixed charge density $\Sigma(\mathbf{r})$ and can be written as

$$
\mathscr{Q}(\mathbf{r})=\sum_{k \alpha} Q\left(\mathbf{r}-\mathbf{R}_{k \alpha}, \boldsymbol{\Omega}_{k \alpha}\right)+\Sigma(\mathbf{r})
$$

where $\mathbf{R}_{k \alpha}$ is the position of the $k$ th particle of type $\alpha$, and $\boldsymbol{\Omega}_{k \alpha}$ denotes its orientation.

Charges are sources for the electrostatic potential $\phi(\mathbf{r})$. These two quantities are related by the Poisson equation [1]:

$$
-\frac{1}{4 \pi} \nabla \cdot[\varepsilon(\mathbf{r}) \nabla \phi(\mathbf{r})]=\mathscr{Q}(\mathbf{r}) .
$$

Key to the description of electrostatic interactions is the Green's function $G_{0}$ of the Poisson equation, which is defined through [1]

$$
-\frac{1}{4 \pi} \nabla \cdot\left[\varepsilon(\mathbf{r}) \nabla G_{0}\left(\mathbf{r}, \mathbf{r}^{\prime}\right)\right]=\delta^{d}\left(\mathbf{r}-\mathbf{r}^{\prime}\right),
$$

The Green's function $G_{0}\left(\mathbf{r}, \mathbf{r}^{\prime}\right)$ gives the electrostatic potential $\phi(\mathbf{r})$ generated at position $\mathbf{r}$ due to a point charge located at $\mathbf{r}^{\prime}$.In the specific case where the dielectric constant has a uniform value $\varepsilon$ through the entire system, the explicit form of the Green's function, denoted by $G_{\text {free }}$, is

$$
G_{\text {free }}\left(\mathbf{r}, \mathbf{r}^{\prime}\right)=\frac{1}{\varepsilon\left|\mathbf{r}-\mathbf{r}^{\prime}\right|} .
$$

The form of the Green's function is affected by the variations in the dielectric constant $\varepsilon(\mathbf{r})$ through the system, which reflects the polarizability of the background. 
The manner in which the potential emanates from a general charge distribution and propagates through the system is characterized by the Green's function:

$$
\phi(\mathbf{r})=\int d \mathbf{r}^{\prime} G_{0}\left(\mathbf{r}, \mathbf{r}^{\prime}\right) \mathscr{Q}\left(\mathbf{r}^{\prime}\right)
$$

which is simply another manner to write the Poisson equation.

The total electrostatic energy $E^{\text {elec }}$ of the system can be written as the product of the charge density in the system and the electrostatic potential [1]:

$$
E^{\mathrm{elec}}=\frac{1}{2} \int d \mathbf{r} \mathscr{Q}(\mathbf{r}) \phi(\mathbf{r}) .
$$

Substituting the expression for the electrostatic potential in terms of the charge density, as given in Eq. (5), the energy of the electrostatic field can be written in a more familiar form:

$$
E^{\text {elec }}=\frac{1}{2} \int d \mathbf{r} d \mathbf{r}^{\prime} \mathscr{Q}(\mathbf{r}) G_{0}\left(\mathbf{r}, \mathbf{r}^{\prime}\right) \mathscr{Q}\left(\mathbf{r}^{\prime}\right)
$$

This expression for the energy generalizes Coulomb's law (where $G_{0}$ is replaced with $G_{\text {free }}$ ) for the situation where the dielectric constant is not spatially uniform dielectric constant.

The expression for the electrostatic energy given in Eq. (7) contains contributions from the interaction of the charge of a particle with the electrostatic potential that it generates - the self energy of the particle. The electrostatic self energy of a particle of type $\alpha$ is given by

$$
e_{\alpha}^{\mathrm{se}}(\mathbf{R}, \boldsymbol{\Omega})=\frac{1}{2} \int d \mathbf{r} d \mathbf{r}^{\prime} Q_{\alpha}(\mathbf{r}-\mathbf{R}, \boldsymbol{\Omega}) G_{\text {free }}\left(\mathbf{r}, \mathbf{r}^{\prime}\right) Q_{\alpha}\left(\mathbf{r}^{\prime}-\mathbf{R}, \boldsymbol{\Omega}\right)
$$

The self energy is the interaction of a particle's charge distribution with the electrostatic potential it generates in the absence of spatial variations of the background dielectric constant. The introduction of inhomogeneities in the dielectric constant, such as polarizable bodies or dielectric interfaces will lead to a shift of the self energy.

Removing this self energy contribution (which is potentially infinite, such as for a point charge), the electrostatic interaction energy between particles is given by

$$
E^{\mathrm{elec}}=\frac{1}{2} \int d \mathbf{r} d \mathbf{r}^{\prime} \mathscr{Q}(\mathbf{r}) G_{0}\left(\mathbf{r}, \mathbf{r}^{\prime}\right) \mathscr{Q}\left(\mathbf{r}^{\prime}\right)-\sum_{k \alpha} e_{\alpha}^{\mathrm{se}}\left(\mathbf{R}_{k \alpha}, \boldsymbol{\Omega}_{k \alpha}\right) .
$$

\subsection{Partition function}

All the equilibrium static properties (e.g., structural and thermodynamic) of an open system of fixed volume $V$, temperature $T$, and species chemical potential $\mu_{\alpha}$ can be determined from knowledge of the grand partition function $Z_{G}$. The grand partition 
function of this system is given by [2]

$$
\begin{aligned}
Z_{G}[\gamma, \Sigma]= & \sum_{N_{1}=0}^{\infty} \cdots \sum_{N_{M}=0}^{\infty} \prod_{v} \frac{1}{N_{v} ! \Lambda_{v}^{d N_{v}}} \\
& \times \int \prod_{t \tau} d \mathbf{R}_{t \tau} d \boldsymbol{\Omega}_{t \tau} e^{-\beta E^{\mathrm{elec}}-\beta E^{\mathrm{ref}}+\sum_{k \alpha} \gamma_{\alpha}\left(\mathbf{R}_{k \alpha}, \boldsymbol{\Omega}_{k \alpha}\right)}
\end{aligned}
$$

where $\beta=1 /\left(k_{B} T\right), k_{B}$ is the Boltzmann constant, $\Lambda_{\alpha}$ is the de Broglie wavelength of particles of type $\alpha, \gamma_{\alpha}\left(\mathbf{R}_{k \alpha}, \boldsymbol{\Omega}_{k \alpha}\right)=\beta \mu_{\alpha}-\beta u_{\alpha}^{(1)}\left(\mathbf{R}_{k \alpha}, \boldsymbol{\Omega}_{k \alpha}\right)$ is a dimensionless chemical potential which includes the effect of an applied one-body potential $u_{\alpha}^{(1)}$, and $E^{\text {ref }}$ is the interaction energy between particles that is not due to electrostatics (e.g., excluded volume).

In order to transform the partition function from a sum over particle positions and orientations to a sum over shapes of a fluctuating field, we introduce the HubbardStratonovich transformation $[3,4]$ in order to rewrite the grand partition function of the system as a functional integral. In terms of the electrolyte systems, this approach has been used by many researchers $[5,6,7,8]$. This transformation allows us to represent the electrostatic interaction energy $E^{\text {elec }}$ of the charge distribution in the system in terms of the following average

$$
\exp \left(-\frac{1}{2 \beta} \int d \mathbf{r} d \mathbf{r}^{\prime} \mathscr{Q}(\mathbf{r}) G_{0}\left(\mathbf{r}, \mathbf{r}^{\prime}\right) \mathscr{Q}\left(\mathbf{r}^{\prime}\right)\right)=\left\langle e^{-\int d \mathbf{r} \mathscr{Q}(\mathbf{r}) i \psi(\mathbf{r})}\right\rangle_{0}
$$

where

$$
\langle(\cdots)\rangle_{0}=\frac{1}{\mathscr{N}_{0}} \int \mathscr{D} \psi(\cdot)(\cdots) e^{-H_{0}[\psi]},
$$

the Hamiltonian $H_{0}$ is given by

$$
H_{0}[\psi]=\frac{1}{2 \beta} \int d \mathbf{r} d \mathbf{r}^{\prime} \psi(\mathbf{r}) G_{0}^{-1}\left(\mathbf{r}, \mathbf{r}^{\prime}\right) \psi\left(\mathbf{r}^{\prime}\right)
$$

and $\mathscr{N}_{0}$ is a normalization constant which is given by

$$
\mathscr{N}_{0}=\int \mathscr{D} \psi(\cdot) e^{-H_{0}[\psi]} \propto\left(\operatorname{det} G_{0}^{-1}\right)^{1 / 2}
$$

Consequently, we find that the grand partition function can be written as

$$
Z_{G}[\gamma, \Sigma]=\left\langle Z_{G}^{\mathrm{ref}}\left[\gamma-Q i \psi+\beta e^{\mathrm{se}}\right] \exp \left[-\int d \mathbf{r} \Sigma(\mathbf{r}) i \psi(\mathbf{r})\right]\right\rangle_{0}
$$

where $Z_{G}^{\text {ref }}[\gamma]$ is the grand partition function of a system where there are no electrostatic interactions between the particles.

The main physical interpretation of this mathematical transformation of the partition function is that the grand partition function of a system with electrostatic interactions is identical to a system without electrostatic interactions, but with each 
particle interacting with a "randomly" fluctuating external field. The strength of the coupling of each particle to this field is proportional to its charge:

$$
\gamma_{\alpha}(\mathbf{R}, \boldsymbol{\Omega}) \rightarrow \gamma_{\alpha}(\mathbf{R}, \boldsymbol{\Omega})-\int d \mathbf{r} Q_{\alpha}(\mathbf{r}-\mathbf{R}, \boldsymbol{\Omega}) i \psi(\mathbf{r})+\beta e_{\alpha}^{\mathrm{se}}(\mathbf{R}, \boldsymbol{\Omega}) .
$$

The field $\psi$ is not entirely randomly distributed but, rather, is spatially correlated to itself. It is actually distributed according to a Gaussian probability distribution function with a zero mean. The correlation of a fluctuation of $\psi$ at a position $\mathbf{r}$ and a fluctuation at a position $\mathbf{r}^{\prime}$ is given by the Green's function of the Poisson equation (i.e. $\left\langle\psi(\mathbf{r}) \psi\left(\mathbf{r}^{\prime}\right)\right\rangle=\beta G_{0}\left(\mathbf{r}, \mathbf{r}^{\prime}\right)$ ). The Hubbard-Stratonovich transformation is not limited to electrostatic interactions, but it can also be performed on any general pairwise additive interaction. The two-point correlation of the fluctuating field is proportional to the pairwise interaction potential of the system.

\subsection{Dispersion interactions}

Before we discuss methods to evaluate the grand partition function, there are a couple of nice features that should be mentioned about the functional integral formulation. First, the "fluctuations" of the electric potential in the system were assumed to be due to the thermal motion of the ions in the system. However, we can consider the partition function for a system without any charged particles. The partition function essentially becomes the determinant of the Green's function of the Poisson equation. Changes in the arrangement of dielectric bodies in the system will alter the function $\varepsilon \mathbf{r}$ ), and, consequently, will affect the Green's function $G_{0}(\mathbf{r}, \mathbf{r})$ of the Poisson equation. This will lead to differences in the normalization constant $\mathscr{N}$ of the functional integral, which are essentially related to the determinant of the Green's function

$$
\beta F=-\ln \frac{\mathscr{N}_{0}}{\mathscr{N}_{\text {free }}}=\frac{1}{2} \operatorname{Tr} \ln \frac{G_{0}}{G_{\text {free }}} .
$$

Interestingly, in this case, the functional integral formulation reduces to the Lifshitz theory of dispersion interaction [9]. The theory is able to nicely couple dispersion (aka van der Waals) forces and electrostatic interactions within a single framework [10].

The difference in this determinant in the situation where there are dielectric bodies versus the absence of dielectric bodies (the free state), leads to an effective interaction between the bodies due to shift in the normal modes available to the electric potential. So one nice thing about this transformation is that it allows us to naturally couple the thermal motion of the charged particles in the system to the zero frequency dispersion interactions. For example, we naturally get screening of the dispersion interaction $[7,11,12,13]$. 


\subsection{Mean-field approximation}

The functional integral formulation of the partition function is exact. Unfortunately, it is not possible to analytically evaluate the functional integral due to the nonlinear dependence of the reference partition function $\ln Z^{\text {ref }}$ on the one-body potentials $\gamma_{\alpha}(\mathbf{R}, \boldsymbol{\Omega})$. As with all exact truths, we really can not do anything useful until we make some sort of approximation or simplifying assumption.

One of the simplest approximations is to neglect the fluctuations in the field altogether and simply choose the value of the field which has the "largest" contribution to the free energy - the largest value of the integrand. That is, we approximate the average of a quantity of a functional $A[\psi]$ as

$$
\left\langle e^{A[\psi]}\right\rangle_{0} \approx e^{A[\bar{\psi}]}
$$

where $\bar{\psi}$ is the form of the function $\psi$ which makes the functional $e^{A[\psi]}$ stationary:

$$
\frac{\delta A[\bar{\psi}]}{\delta \psi(\mathbf{r})}=0
$$

This is known as the mean field approximation.

Applying this to the evaluation of the grand partition function, we find

$$
\begin{aligned}
\ln Z_{G}[\gamma, \Sigma] \approx & \frac{1}{2 \beta} \int d \mathbf{r} d \mathbf{r}^{\prime} i \bar{\psi}(\mathbf{r}) G_{0}^{-1}\left(\mathbf{r}, \mathbf{r}^{\prime}\right) i \bar{\psi}(\mathbf{r})-\int d \mathbf{r} \Sigma(\mathbf{r}) i \bar{\psi}(\mathbf{r}) \\
& +\ln Z_{G}^{\operatorname{ref}}[\gamma-Q i \bar{\psi}]
\end{aligned}
$$

The final term is the grand potential of the reference system, where the chemical potential (or one-body potential) of the particles has been shifted by a coupling of the particle by its charge to the mean field $\bar{\psi}$ :

$$
\gamma_{\alpha}(\mathbf{R}, \boldsymbol{\Omega}) \rightarrow \gamma_{\alpha}(\mathbf{R}, \boldsymbol{\Omega})-\int d \mathbf{r} Q_{\alpha}(\mathbf{r}-\mathbf{R}, \boldsymbol{\Omega}) i \bar{\psi}(\mathbf{r})
$$

In the mean field approximation, contributions of configurations of the system that deviate from the representative value $\bar{\psi}$ are entirely neglected.

The value of $\bar{\psi}$ is determined by the stationary condition

$$
\frac{\delta \ln Z_{G}[\gamma, \Sigma]}{\delta \bar{\psi}(\mathbf{r})}=0
$$

This leads to the Poisson equation:

$$
-\frac{1}{4 \pi \beta} \nabla \cdot \varepsilon(\mathbf{r}) \nabla i \bar{\psi}(\mathbf{r})=\sum_{\alpha} \int d \mathbf{R} d \boldsymbol{\Omega} Q_{\alpha}(\mathbf{r}-\mathbf{R}, \boldsymbol{\Omega}) \rho_{\alpha}(\mathbf{R}, \boldsymbol{\Omega})+\Sigma(\mathbf{r})
$$


where $\rho_{\alpha}(\mathbf{R}, \boldsymbol{\Omega})$ density of particles of type $\alpha$ in the system. From this, we find that the field $i \bar{\psi}$ can be directly related to the average electrostatic potential in the system as $i \bar{\psi}(\mathbf{r})=\beta \phi(\mathbf{r})$.

The particle density is given by

$$
\rho_{\alpha}(\mathbf{R}, \boldsymbol{\Omega})=\rho_{\alpha}^{\mathrm{ref}}(\mathbf{R}, \boldsymbol{\Omega} ; \gamma-Q i \bar{\psi}) .
$$

Within the mean-field approximation, we see that the particle density is the same as for the reference system (which does not include electrostatic interactions) but with an additional one-body interaction given by the coupling of the particle charge to the average electrostatic potential [14].

This is a good approximation when the charge of the particles and the magnitude of any fixed charges are relatively small. However, if either of these become significant (e.g., multivalent ion or surfaces with a high charge density), then fluctuations make an important contribution to the properties of the system. There are also situations where even though the fluctuations in a system may be small, they can lead to qualitative differences in the predictions of the theory. One such example is the depletion of ions in aqueous solution from an air-water interface, thereby increasing the interfacial tension.

One manner to incorporate fluctuations in the evaluation of the functional integral is to expand the Hamiltonian in terms of fluctuations in the field around the mean field value. This is known as the loop approximation. When truncated at quadratic order, this is the one-loop approximation. This type of approach has been performed analytically $[7,11,15]$ and numerically (e.g., lattice field theory methods $[6,16,17])$. by many authors. One difficulty with the loop expansion is that it leads to divergences, which must be dealt with by careful renormalization of the theory. In the following section, we will present another approach, known as the variational perturbation approximation, that is free of this issue and has many other nice features.

\section{Variational perturbation approximation}

In the previous section, we mentioned mean field theory, where the functional integral is approximated by only one representative distribution of the field $\bar{\psi}$. So long as the contributions of other shapes of the field are much smaller, then the mean field approximation should be good. In this section, we discuss the variational perturbation approximation [18], a method to systematically include fluctuation contributions to the partition function to increasing orders of accuracy.

In this approach, the integrand in the partition function (see Eq. (15)) is approximated with a Gaussian function:

$$
H_{\mathscr{K}}[\psi]=\frac{1}{2 \beta} \int d \mathbf{r} d \mathbf{r}^{\prime}[\psi(\mathbf{r})-\bar{\psi}(\mathbf{r})] G_{\mathscr{K}}^{-1}\left(\mathbf{r}, \mathbf{r}^{\prime}\right)\left[\psi\left(\mathbf{r}^{\prime}\right)-\bar{\psi}\left(\mathbf{r}^{\prime}\right)\right]
$$


where $G_{\mathscr{K}}$ is a renormalized Green's function, defined as

$$
G_{\mathscr{K}}^{-1}\left(\mathbf{r}, \mathbf{r}^{\prime}\right)=G_{0}^{-1}\left(\mathbf{r}, \mathbf{r}^{\prime}\right)+\mathscr{K}\left(\mathbf{r}, \mathbf{r}^{\prime}\right) .
$$

The grand partition function is then rewritten in terms of averages with respect to the variational Hamiltonian $H_{\mathscr{K}}$ as

$$
\ln Z_{G}[\gamma, \Sigma]=\left\langle e^{\ln Z_{G}^{\mathrm{ref}}\left[\gamma-i Q \psi+\beta e^{\mathrm{se}}\right]-\left(H[\psi]-H_{\mathscr{K}}[\psi]\right)}\right\rangle_{\mathscr{K}} \frac{\mathscr{N}_{\mathscr{K}}}{\mathscr{N}_{0}}
$$

where $\langle(\cdots)\rangle_{\mathscr{K}}$ denotes an average with respect to the Hamiltonian $H_{\mathscr{K}}$, and $\mathscr{N}_{\mathscr{K}}$ is the associated normalization constant. The ratio which follows the average is the change of normalization constant due to the change of the average from using $H_{0}$ to using $H_{\mathscr{K}}$.

The average of the exponential term in Eq. (26) can be evaluated using a cumulant expansion:

$$
\ln \left\langle e^{A[\psi]}\right\rangle \approx\langle A[\psi]\rangle^{(c)}+\frac{1}{2 !}\left\langle A^{2}[\psi]\right\rangle^{(c)}+\frac{1}{3 !}\left\langle A^{3}[\psi]\right\rangle^{(c)}+\cdots
$$

where $\left\langle A^{n}[\psi]\right\rangle^{(c)}$ denotes the $n$th cumulant of the distribution. Truncating this expansion to first order, we find the following inequality

$$
\begin{aligned}
\ln Z_{G}[\gamma, \Sigma] & \geq \int d \mathbf{r} d \mathbf{r}^{\prime} i \bar{\psi}(\mathbf{r}) G_{0}^{-1}\left(\mathbf{r}, \mathbf{r}^{\prime}\right) i \bar{\psi}\left(\mathbf{r}^{\prime}\right)-\int d \mathbf{r} \Sigma(\mathbf{r}) i \bar{\psi}(\mathbf{r}) \\
& +\left\langle\ln Z_{G}^{\mathrm{ref}}\left[\gamma-i Q \psi+\beta e^{\mathrm{se}}\right]\right\rangle_{\mathscr{K}}+\frac{1}{2} \operatorname{Tr} \mathscr{K} G_{\mathscr{K}}+\ln \frac{\mathscr{N}_{\mathscr{K}}}{\mathscr{N}_{0}}
\end{aligned}
$$

The true value of the grand partition function will be larger than estimate of the first order cumulant approximation for any choice of $\bar{\psi}$ and $\mathscr{K}$.

In the exact theory, the predictions for the properties of the system are independent of the choice of $\bar{\psi}$ and $\mathscr{K}$; however, by making approximations, the resulting theory will have a dependence on these quantities. In order to minimize this effect, select these values such that the free energy is stationary with respect to variations in these quantities. This leads to the following variation conditions:

$$
\begin{aligned}
\frac{\delta F}{\delta \bar{\psi}(\mathbf{r})} & =0 \\
\frac{\delta F}{\delta \mathscr{K}\left(\mathbf{r}, \mathbf{r}^{\prime}\right)} & =0 .
\end{aligned}
$$

These conditions can be used to determine the "optimal" values of the quantities $\bar{\psi}$ and $\mathscr{K}$.

Equation (28) leads to the Poisson equation

$$
-\frac{1}{4 \pi \beta} \nabla \cdot \varepsilon(\mathbf{r}) \nabla i \bar{\psi}(\mathbf{r})=\sum_{\alpha} \int d \mathbf{R} d \boldsymbol{\Omega} Q_{\alpha}(\mathbf{r}-\mathbf{R}, \boldsymbol{\Omega}) \rho_{\alpha}(\mathbf{R}, \boldsymbol{\Omega})+\Sigma(\mathbf{r})
$$


where $\rho_{\alpha}(\mathbf{R}, \boldsymbol{\Omega})$ is the density of particles of type $\alpha$ and is given by

$$
\rho_{\alpha}(\mathbf{R}, \boldsymbol{\Omega})=\left\langle\rho_{\alpha}^{\mathrm{ref}}\left(\mathbf{R}, \boldsymbol{\Omega} ; \boldsymbol{\gamma}-Q i \psi+\beta e^{\mathrm{se}}\right)\right\rangle_{\mathscr{K}} .
$$

From this relation, we see that $\bar{\psi}(\mathbf{r})=\beta \phi(\mathbf{r})$ can be identified with the average of the electrostatic potential, as in the mean-field approximation.

The variational relation with respect to the screening function $\mathscr{K}$ (see Eq. (29)) leads to

$$
\begin{aligned}
\mathscr{K}\left(\mathbf{r}, \mathbf{r}^{\prime}\right)= & \left\langle\psi(\mathbf{r}) \psi\left(\mathbf{r}^{\prime}\right) \ln Z_{G}^{\mathrm{ref}}\left[\gamma-Q i \psi+\beta e^{\mathrm{se}}\right]\right\rangle_{\mathscr{K}} \\
& -G_{\mathscr{K}}\left(\mathbf{r}, \mathbf{r}^{\prime}\right)\left\langle\ln Z_{G}^{\mathrm{ref}}\left[\gamma-Q i \psi+\beta e^{\mathrm{se}}\right]\right\rangle_{\mathscr{K}} .
\end{aligned}
$$

One of the key challenges to applying this theory is the ability to solve this equation. In particular, it is typically quite difficult to determine a closed, analytical form for $G_{\mathscr{K}}\left(\mathbf{r}, \mathbf{r}^{\prime}\right)$. One approximation strategy is to find some simple variational form for $\mathscr{K}\left(\mathbf{r}, \mathbf{r}^{\prime}\right)$ where the Green's function is known, and then vary the parameters of this function to minimize the free energy.

The Helmholtz free energy functional can be determined by performing a Legendre transform:

$$
F[\rho, \Sigma]=\sum_{\alpha} \int d \mathbf{R} d \boldsymbol{\Omega} \rho_{\alpha}(\mathbf{R}, \boldsymbol{\Omega}) \gamma_{\alpha}(\mathbf{R}, \boldsymbol{\Omega})-\ln Z_{G}[\gamma, \Sigma] .
$$

The resulting expression for the free energy is:

$$
\begin{aligned}
F[\rho, \Sigma] & =\sum_{\alpha} \int d \mathbf{R} d \boldsymbol{\Omega} \rho_{\alpha}(\mathbf{R}, \boldsymbol{\Omega})\left[\ln \rho_{\alpha}(\mathbf{R}, \boldsymbol{\Omega}) \Lambda_{\alpha}^{d}-1\right] \\
& -\frac{1}{2 \beta} \int d \mathbf{r} d \mathbf{r}^{\prime} i \bar{\psi}(\mathbf{r}) G_{0}^{-1}\left(\mathbf{r}, \mathbf{r}^{\prime}\right) i \bar{\psi}\left(\mathbf{r}^{\prime}\right) \\
& +\int d \mathbf{r}\left[\int d \mathbf{R} d \boldsymbol{\Omega} \rho_{\alpha}(\mathbf{R}, \boldsymbol{\Omega}) Q_{\alpha}(\mathbf{r}-\mathbf{R}, \boldsymbol{\Omega})+\Sigma(\mathbf{r})\right] i \bar{\psi}(\mathbf{r}) \\
& -\ln \frac{\mathscr{N}_{\mathscr{K}}}{\mathscr{N}_{0}}-\frac{1}{2} \operatorname{Tr} \mathscr{K} G_{0} .
\end{aligned}
$$

From the free energy functional, we can derive all static equilibrium properties of the system.

The use of the variational approximation assumes that the fluctuations of the field are weak and can be represented by a Gaussian distribution. While this is a good approximation for fluctuations over large length scales, at short wavelengths it breaks down. 


\section{Point charge model}

To concretely illustrate the application of this theoretical framework, we consider a system of point charges. This model is commonly used to describe electrolyte systems. The charge density for a point charge is given by

$$
Q_{\alpha}(\mathbf{r}, \boldsymbol{\Omega})=q_{\alpha} \delta^{d}(\mathbf{r})
$$

Half of the particles are cations (denoted by + ) with charge $q$, and the other half are anions (denoted by - ) with charge $-q$.

There are no other interactions in the system apart from electrostatic interactions. The reference system is, therefore, an ideal gas, which has a grand partition function given by

$$
Z_{G}[\gamma]=\sum_{\alpha} \Lambda_{\alpha} \int d \mathbf{R} d \boldsymbol{\Omega} e^{\gamma_{\alpha}(\mathbf{R}, \boldsymbol{\Omega})}
$$

A key length scale for this system is the Bjerrum length $l_{B}=\beta q^{2} / \varepsilon$, which is the distance at which the electrostatic interaction energy between two charges equals their thermal energy $k_{B} T$.

\subsection{Mean-field approximation}

We first examine the mean-field approximation for the point charge system. The grand partition function for the point charge model in the mean field approximation is given by

$$
\begin{gathered}
\ln Z_{G}[\gamma, \Sigma] \approx \frac{1}{2 \beta} \int d \mathbf{r} d \mathbf{r}^{\prime} i \bar{\psi}(\mathbf{r}) G_{0}^{-1}\left(\mathbf{r}, \mathbf{r}^{\prime}\right) i \bar{\psi}\left(\mathbf{r}^{\prime}\right)-\int d \mathbf{r} \Sigma(\mathbf{r}) i \bar{\psi}(\mathbf{r}) \\
+\sum_{\alpha} \Lambda_{\alpha}^{d} \int d \mathbf{R} e^{\gamma_{\alpha}(\mathbf{R})-q_{\alpha} i \bar{\psi}(\mathbf{R})}
\end{gathered}
$$

The value of the mean field is determined from the stationary condition, given in

$$
-\frac{1}{4 \pi \beta} \nabla \cdot \varepsilon(\mathbf{r}) \nabla i \bar{\psi}(\mathbf{r})=\sum_{\alpha} q_{\alpha} \rho_{\alpha}(\mathbf{r})+\Sigma(\mathbf{r})
$$

where $\rho_{\alpha}(\mathbf{r})$ is the density of particles of type $\alpha$.

From the grand partition function, we can derive all static equilibrium properties of the system. In particular, the density of particles of type $\alpha$ is give by:

$$
\rho_{\alpha}(\mathbf{R})=\Lambda_{\alpha}^{d} e^{\gamma_{\alpha}(\mathbf{R})-q_{\alpha} i \bar{\psi}(\mathbf{R})} .
$$

Therefore, we see that the mean field approximation leads to the Poisson-Boltzmann theory. 
The corresponding expression for the Helmholz free energy is

$$
\begin{aligned}
F[\rho, \Sigma] \approx- & \frac{1}{2 \beta} \int d \mathbf{r} d \mathbf{r}^{\prime} i \bar{\psi}(\mathbf{r}) G_{0}^{-1}\left(\mathbf{r}, \mathbf{r}^{\prime}\right) i \bar{\psi}(\mathbf{r})+\int d \mathbf{r}\left[\sum_{\alpha} q_{\alpha} \rho_{\alpha}(\mathbf{r})+\Sigma(\mathbf{r})\right] i \bar{\psi}(\mathbf{r}) \\
& +\sum_{\alpha} \int d \mathbf{R} \rho_{\alpha}(\mathbf{R})\left[\ln \rho_{\alpha}(\mathbf{R}) \Lambda_{\alpha}^{d}-1\right] .
\end{aligned}
$$

\subsection{Debye-Hückel theory}

Now we apply the variational perturbation theory at first order to the point charge model. First we consider a uniform system. In a uniform system, $\bar{\psi}(\mathbf{r})=0$ due to symmetry. Using this fact, combined with the expression for the renormalized Green's function, the grand partition function is determined to be

$$
\frac{1}{V} \ln Z_{G}[\gamma] \geq \sum_{\alpha} z_{\alpha} e^{\frac{\kappa l_{B}}{2}}-\frac{\kappa^{3}}{24 \pi}
$$

where $V$ is the system volume, and $z_{\alpha}=\Lambda_{\alpha}^{d} e^{\gamma_{\alpha}}$ is the fugacity of ions of type $\alpha$. Due to electroneutrality, the fugacity of the cations and anions must be equal; we denote this common value by $z_{ \pm}$.

From the variational condition (see Eq. (29)), the renormalized Green's function is

$$
G_{\mathscr{K}}\left(\mathbf{r}, \mathbf{r}^{\prime}\right)=\frac{e^{-\kappa\left|\mathbf{r}-\mathbf{r}^{\prime}\right|}}{\varepsilon\left|\mathbf{r}-\mathbf{r}^{\prime}\right|}
$$

where $\kappa$ is inverse Debye screening length

$$
\kappa^{2}=\frac{4 \pi \beta}{\varepsilon} \sum_{\alpha} q_{\alpha}^{2} \rho_{\alpha}=8 \pi \rho_{ \pm}
$$

where $\rho_{ \pm}$is the electrolyte concentration. Accounting for the fluctuations of the electrostatic potential due to the thermal motion of the point charges leads to an exponential decay of the Green's function. Physically, this corresponds to screening of charge.

The Helmholtz free energy is

$$
F[\rho, \Sigma] \approx+V \sum_{\alpha} \rho_{\alpha}\left(\ln \rho_{\alpha} \Lambda_{\alpha}^{d}-1\right)-V \frac{\kappa^{3}}{24 \pi} .
$$

The chemical potential of species $\alpha$ is given by

$$
\beta \mu_{\alpha}=\ln \rho_{\alpha} \Lambda_{\alpha}^{d}-\frac{\beta q_{\alpha}^{2} \kappa}{2 \varepsilon}
$$


The first order variational perturbation approximation for point charges leads to the Debye-Hückel theory of electrolyte solutions.

For a nonuniform systems, the Helmholtz free energy is:

$$
\begin{aligned}
F[\rho, \Sigma] \approx- & \frac{1}{2 \beta} \int d \mathbf{r} d \mathbf{r}^{\prime} i \bar{\psi}(\mathbf{r}) G_{0}^{-1}\left(\mathbf{r}, \mathbf{r}^{\prime}\right) i \bar{\psi}\left(\mathbf{r}^{\prime}\right)+\int d \mathbf{r}\left[\sum_{\alpha} q_{\alpha} \rho_{\alpha}(\mathbf{r})+\Sigma(\mathbf{r})\right] i \bar{\psi}(\mathbf{r}) \\
& +\sum_{\alpha} \int d \mathbf{R} \rho_{\alpha}(\mathbf{R})\left[\ln \rho_{\alpha}(\mathbf{R}) \Lambda_{\alpha}^{d}-1\right]-\ln \frac{\mathscr{N}_{\mathscr{K}}}{\mathscr{N}_{0}}-\frac{1}{2} \operatorname{Tr} \mathscr{K} G_{0} .
\end{aligned}
$$

In this case the screening function becomes local

$$
\mathscr{K}\left(\mathbf{r}, \mathbf{r}^{\prime}\right)=\delta^{d}\left(\mathbf{r}-\mathbf{r}^{\prime}\right) \sum_{\alpha} q_{\alpha}^{2} \rho_{\alpha}(\mathbf{r})
$$

We can identify $\mathscr{K}$ with the inverse Debye screening length $\kappa$, which varies with the local density

$$
\kappa^{2}(\mathbf{r})=\frac{4 \pi \beta}{\varepsilon} \sum_{\alpha} q_{\alpha}^{2} \rho_{\alpha}(\mathbf{r})
$$

For a uniform system, this becomes a constant.

Before, we mentioned that there is a "self-energy" of the particles, due to the interaction of each charge with the electrical potential that it generates itself. This seemingly irrelevant term, which is infinite for point charges, was subtracted out. However, interestingly, it reappears again in the theory. Due to the screening of charge by the response of the other charged particles in the system, the electric potential that a point charge generates is altered. This shifts the self-energy of the point charge. What we find is that the difference in the self-energy of the point charge in the presence and in the absence of other mobile charges actually is the excess chemical potential of the particle. We can see this term in the expression for the particle density

$$
\rho_{\alpha}(\mathbf{R})=\Lambda_{\alpha}^{d} e^{\gamma_{\alpha}(\mathbf{R})-q_{\alpha} i \bar{\psi}(\mathbf{R})-\frac{\beta q^{2}}{2} \Delta G_{\mathrm{K}}(\mathbf{R}, \mathbf{R})} .
$$

where $\Delta G_{\mathrm{K}}=G_{\mathrm{K}}-\Delta G_{\text {free }}$ is the shift in the Green's function due to the presence of fluctuations. The self energy term changes propensity of a particle to remain at a location due to fluctuations in the local environment caused by thermal motion of charge.

\subsection{Stability of the point charge model}

In the previous section, we demonstrated that the first order variational perturbation approximation leads to the Debye-Hückel theory for electrolyte solutions. In the variational perturbation approximation, the value of the inverse screening length is 


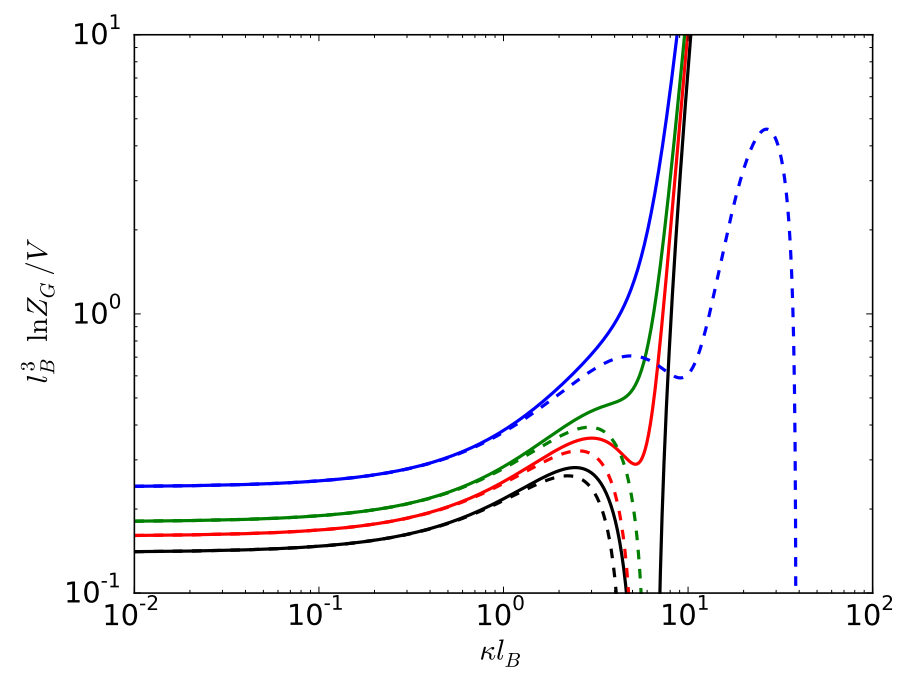

Fig. 1 Stability of the point charge model. (a) Variation of the grand potential with with the inverse screening length $\kappa$ for a fugacity: (i) $z_{ \pm} l_{B}^{3}=0.07$ (black), (ii) $z_{ \pm} l_{B}^{3}=0.08$ (red), and (iii) $z_{ \pm} l_{B}^{3}=0.09$ (green). (iv) $z_{ \pm} l_{B}^{3}=0.12$ (green). The solid lines represent the situation without a cutoff, and the dashed lines are for a cutoff of $\Lambda l_{B}=20$.

determined by maximizing the grand partition function, and this leads to the standard relationship between the inverse screening length and the ionic strength of the system. The astute reader, however, may have noticed that the expression for the grand partition function (see Eq. (41) actually diverges as $\kappa \rightarrow \infty$. From a physical perspective, this is a consequence of the fact that the point charge model is inherently unstable. The positive and negative point charges will collapse with each other, resulting in a situation where there are an infinite number of ions in the system. So then what is the relevance of the Debye-Hückel expression for $\kappa$ ?

To investigate this point, we show the variation of the grand partition function with $\kappa$, given by the solid lines in Fig. 1, for several different values of the electrolyte fugacity $z_{ \pm}$. For all fugacities, $\ln Z_{G}$ is maximized when $\kappa \rightarrow \infty$, indicating that the point charge system wants to collapse. At high fugacities, $\ln Z_{G}$ increases monotonically with increasing $\kappa$. However, for sufficiently low values of $z_{ \pm}$, which corresponds to low ion densities, the grand partition function has a local maximum. This corresponds to the Debye-Hückel theory for the point charge model.

For real electrolytes, excluded volume interactions prevent the collapse of the system. These interactions suppress the fluctuations at a short length scales. Properly accounting for their effect on the grand partition function is quite difficult, however, we can get a qualitative understanding of their influence by introduction a cutoff wavevector $\Lambda$. Fluctuations of length scales less than $2 \pi / \Lambda$ are neglected. The resulting expression for the grand partition function is 


$$
\begin{aligned}
\frac{1}{V} \ln Z_{G}[\gamma] \geq \sum_{\alpha} z_{\alpha} \exp & {\left[\frac{\kappa l_{B}}{2} \frac{2}{\pi} \arctan \frac{\Lambda}{\kappa}\right] } \\
& -\frac{\kappa^{3}}{24 \pi} \frac{2}{\pi}\left[\arctan \frac{\Lambda}{\kappa}-\frac{\Lambda}{\kappa}+\left(\frac{\Lambda}{\kappa}\right)^{3} \ln \left(1+\left(\frac{\kappa}{\Lambda}\right)^{2}\right)\right]
\end{aligned}
$$

This is plotted as the dashed lines in Fig. 1.

Suppressing the short wavelength fluctuations stabilizes the model. The maximum of $\ln Z_{G}$ no longer occurs for $\kappa \rightarrow \infty$; it now occurs for finite values of the inverse screening length for all values of the fugacity. At high electrolyte fugacities, the maximum in the grand partition function is no longer related to the DebyeHückel value. In this situation, the system depends sensitively on the value of $\Lambda$. Its properties are controlled by the physics at short wavelengths. At these conditions, the point charge model is irrelevant and not applicable.

At low electrolyte fugacities, the maximum is close to the Debye-Hückel value, although it is slightly shifted; the lower the fugacity (ion concentration), the nearer this peak is to the Debye-Hückel value. For these conditions, the system is relatively insensitive to the precise value of the cutoff $\Lambda$. In this case, the short wavelength physics are irrelevant, and the point charge model is relevant.

It is also interesting to note that multiple peaks can appear in the dependence of the grand partition function on the inverse screening length. Each peak correspond to a distinct phase of the system. Phase coexistence occurs when the height of the peaks are the same. Consequently, we see that this approach is able to predict the vapor-liquid transition of the electrolyte system [19]. Note, however, the quantitative features of this transition (e.g., critical temperature and density) will depend the details of short length scale physics of the system.

\subsection{Dielectric interfaces}

Variations in the dielectric constant of the background medium will affect the manner in which the electrostatic potential propagates from a charge. Here, we examine the influence of a dielectric interface on the properties of the point charge electrolyte. An electrolyte is immersed in a medium with dielectric constant $\varepsilon$ and confined to remain in the half-space defined by $z>0$. For $z<0$, there is a planar body with dielectric constant $\varepsilon^{\prime}$. A schematic diagram of the system is shown in Fig. 3.

When a charge of magnitude $q$ is place near a planar dielectric discontinuity, the difference in the polarizabilities of the two media leads to an induced charge distribution on the interface. The potential generated by this induced charge can be mathematically represented by a point charge of magnitude $q\left(\varepsilon-\varepsilon^{\prime}\right) /\left(\varepsilon+\varepsilon^{\prime}\right)$, located within the dielectric body (see Fig. 2 the same distance from the interface as the original charge. This is known as an image charge.

Consequently, charges are repelled from a low dielectric surface (i.e. $\varepsilon^{\prime}<\varepsilon$ ), regardless of the sign of the charge. This leads to a electrostatic depletion effect 


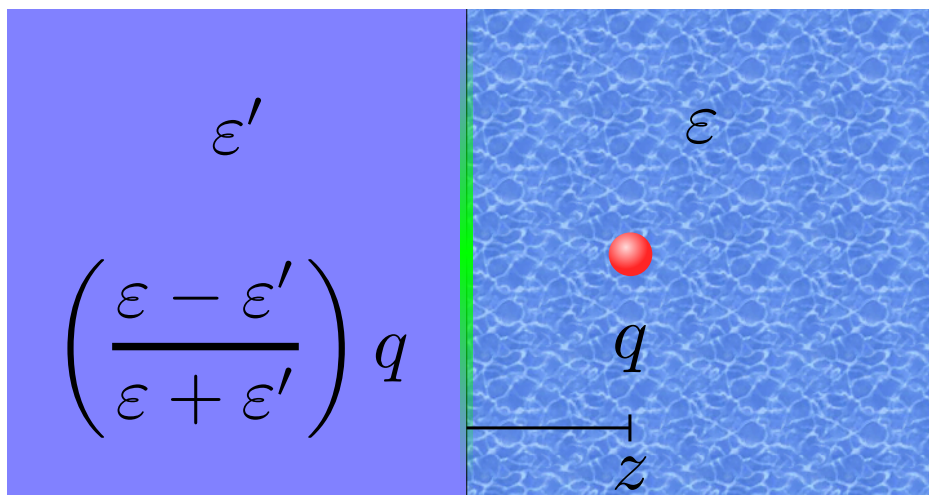

Fig. 2 Schematic diagram of a charge located a distance $z$ from a dielectric interface.

in the case of the low dielectric bodies [20,13,21]. Charges are attracted to high dielectric bodies (i.e. $\varepsilon^{\prime}>\varepsilon$ ), such as metals.

In this planar geometry, the presence of the dielectric interface shifts the Green's function [1]

$$
G_{0}\left(\mathbf{r}, \mathbf{r}^{\prime}\right)=\frac{1}{\varepsilon\left|\mathbf{r}-\mathbf{r}^{\prime}\right|}+\frac{\varepsilon-\varepsilon^{\prime}}{\varepsilon+\varepsilon^{\prime}} \frac{1}{\varepsilon \sqrt{\left(x-x^{\prime}\right)^{2}+\left(y-y^{\prime}\right)^{2}+\left(z+z^{\prime}\right)^{2}}} .
$$

The first term on the right of the equation is the Green's function in a uniform dielectric (i.e. $G_{\text {free }}$ ). The second term is the influence of the dielectric interface and can be interpreted as the potential that eminates from the image charge.

The renormalized Green's function $G_{\mathscr{K}}$ of this system can be determined by solving the variational condition given in Eq. (29). It can be written as the Green's function for the uniform bulk system, where the inverse screening length is equal to $\kappa$, plus a term due to the presence of the dielectric interface:

$$
G_{\mathscr{K}}\left(\mathbf{r}, \mathbf{r}^{\prime}\right)=\frac{e^{-\kappa\left|\mathbf{r}-\mathbf{r}^{\prime}\right|}}{\varepsilon\left|\mathbf{r}-\mathbf{r}^{\prime}\right|}+\delta G_{\mathscr{K}}\left(\mathbf{r}, \mathbf{r}^{\prime}\right)
$$

where $\delta G_{\mathscr{K}}\left(\mathbf{r}, \mathbf{r}^{\prime}\right)$ is shift in the Green's function [20, 22, 23].

If we make the simplifying assumption that the inverse screening length is approximately constant for $z>0$, we find that [20]

$$
\begin{aligned}
\delta G_{\mathscr{K}}(\mathbf{r}, \mathbf{r})= & -\left(\frac{\eta-1}{\eta+1}\right) \frac{e^{-2 \kappa z}}{2 \varepsilon z} \\
& -\frac{2 \eta}{\eta+1} \frac{\kappa}{\varepsilon} \int_{1}^{\infty} d x \frac{\sqrt{x^{2}-1}-x}{\eta \sqrt{x^{2}-1}+x} e^{-2 x \kappa z} .
\end{aligned}
$$



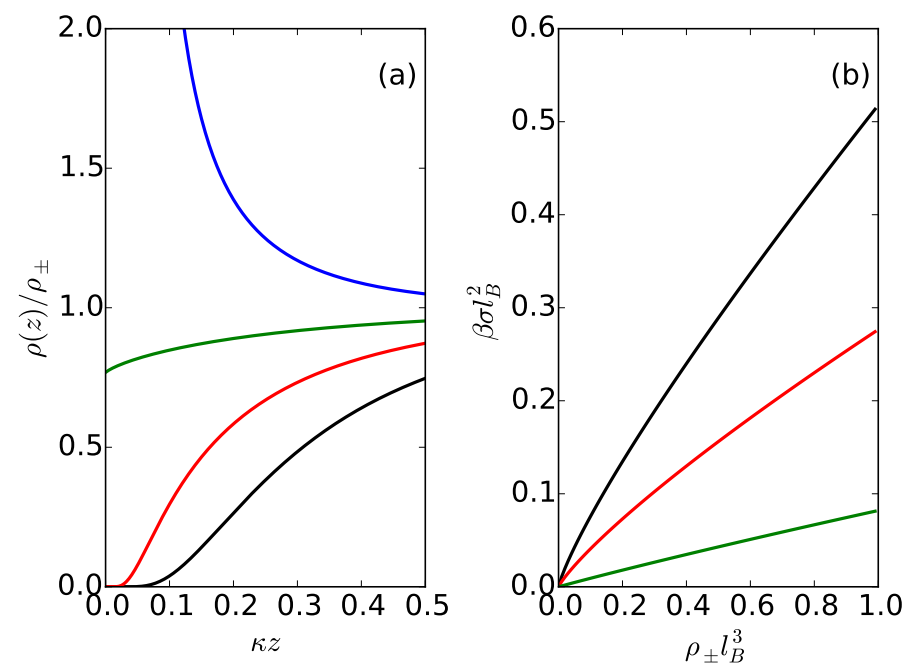

Fig. 3 (a) Ion density profile for an electrolyte solution of concentration $\rho_{ \pm} l_{B}^{3}=0.1$ near a dielectric interface with (i) $\varepsilon^{\prime} / \varepsilon=0$ (black), (ii) $\varepsilon^{\prime} / \varepsilon=0.5$ (red), (iii) $\varepsilon^{\prime} / \varepsilon=1$ (green), and (iv) $\varepsilon^{\prime} / \varepsilon=2$ (blue). (b) Incremental interfacial tension.

The resulting density profiles for the cations $\rho_{+}(\mathbf{r})$ and the anions $\rho_{-}(\mathbf{r})$ are given by

$$
\rho_{+}(\mathbf{r})=\rho_{-}(\mathbf{r})=\rho_{ \pm} \exp \left[-\frac{\beta q^{2}}{2} \delta G_{\mathscr{K}}(\mathbf{r}, \mathbf{r})\right],
$$

where $\rho_{ \pm}$is the ion concentration far from the dielectric interface (i.e. $z \rightarrow \infty$ ). This is plotted in Fig. 3(a) for a system with an electrolyte concentration $\rho_{ \pm} l_{B}^{3}=0.1$ near planar interfaces of various dielectric constants. When the dielectric constant of the interface is larger that that of the solvent (i.e. $\varepsilon^{\prime}>\varepsilon$ ), the ions absorb to the interface. When $\varepsilon^{\prime}<\varepsilon$, such as for electrolytes near an air-water interface, the ions desorb from the interface. Even in the case where there is no dielectric discontinuity (i.e. $\varepsilon^{\prime}=\varepsilon$ ), there is a slight desorption of ions from the interface. This is due to the exclusion of particles from $z<0$ and the preference of charged particles to be fully surrounded by other charged particles.

Adsorption of molecules on an interface will lead to a decrease in the interfacial tension. On the other hand, desorption of solutes from an interface will increase the interfacial tension. This effect is what leads to the increase of the surface tension of water with the addition of electrolytes. For the planar geometry within constant screening approximation, the interfacial tension is given by

$$
\beta \sigma=-\frac{\kappa^{2}}{32 \pi}\left(\frac{\eta-1}{\eta+1}\right)-2 \rho_{ \pm} \int d z\left[e^{-\left(\beta q^{2} / 2\right) \delta G_{\mathscr{K}}(z, z)}-1\right] .
$$


where $\rho_{ \pm}$is the bulk electrolyte concentration. The dependence of the interfacial tension with the bulk electrolyte concentration is presented in Fig. 3(b).

\section{Conclusions}

We have described a field theory approach to the description of electrolyte systems. The evaluation of this theory using the variational perturbation approximation has been discussed. The theory has been developed for particles with a rigid charge distribution. The point charge model is used to illustrate the application of the method. In this case, it was found that the mean field approximation for this system leads to the Poisson-Boltzman theory and the first order variational perturbation approximation leads to the Debye-Hückel theory.

The theoretical framework presented here is quite flexible and applicable to a wide variety of systems and geometries. For example, it has been applied to particles with nonspherically symmetric charge distributions, such as lines [24] and disks [8]. In these cases, the system can form liquid crystalline phases, where the orientations of the molecules are ordered, although the translational degrees of freedom are not. It has also been applied to examine ions confined within pores [13, 22, 23].

When truncated at first order, the variational perturbation approximation is able to go beyond the Poisson-Boltzmann theory, allowing it to capture phenomena like the influence of dielectric interfaces and the coupling between dispersion and electrostatic interactions (i.e. screening of the dispersion). However, it is only accurate for systems where the electrostatic interactions are relatively weak (e.g., low surface charge densities, low ion valencies, and high temperatures). This is due to the assumption that the fluctuations in a system can be well represented by a Gaussian probability distribution, which is good for long wavelength fluctuations but breaks down at short wavelengths. As a consequence, it cannot capture effects that occur at high charge densities, such as overcharging. In principle, the approximation can be extended to higher orders to increase its range of applicability to higher electrostatic coupling strengths; however, the rate of improvement of this expansion is relatively slow.

Another approach to improving the theory is to divide the long and short wavelength fluctuations and treat each with a separate approximation scheme: the variational perturbation approximation for long wavelengths and a virial expansion, for example, for the short wavelength fluctuations. This splitting strategy has been found to be successful in describing electrolyte systems over a broad range of conditions [25, 26], from the weak to the intermediate and including the strong electrostatic coupling regime. This "splitting" approach is able to quantitatively describe phenomena such as charge inversion and like-charge attraction. 


\section{References}

1. J.D. Jackson, Classical Electrodynamics (Wiley, New York, 1975)

2. T.L. Hill, Statistical Mechanics: Principles and Selected Applications (McGraw-Hill, New York, 1956)

3. R.L. Stratonovich, Dokl. Akad. Nauk SSSR 115(6), 1097 (1957)

4. J. Hubbard, Phys. Rev. Lett. 3, 77 (1959). DOI 10.1103/PhysRevLett.3.77

5. A.L. Kholodenko, A.L. Beyerlein, Phys. Rev. A 43(4), 3309 (1986)

6. R.D. Coalson, A. Duncan, J. Chem. Phys. 97(8), 5653 (1992). DOI 10.1063/1.463950

7. R.R. Netz, Phys. Rev. E 60(3), 3174 (1999)

8. L. Lue, Fluid Phase Equil. 241, 236 (2006). DOI 10.1016/j.fluid.2005.11.007

9. J. Mahanty, B.W. Ninham, Dispersion forces (Academic Press, London, 1976)

10. M.M. Hatlo, L. Lue, Soft Matter 4, 1582 (2008). DOI 10.1039/B803783C

11. R.R. Netz, Eur. Phys. J. E 3(2), 131 (2000)

12. R.R. Netz, Eur. Phys. J. E 5, 189 (2001)

13. M.M. Hatlo, R.A. Curtis, L. Lue, J. Chem. Phys. 128(16), 164717 (2008). DOI $10.1063 / 1.2908738$

14. L. Lue, N. Zoeller, D. Blankschtein, Langmuir 15(11), 3726 (1999). DOI 10.1021/la9813376

15. D.S. Dean, R.R. Horgan, Phys. Rev. E 69(6), 061603 (2004). DOI 10.1103/PhysRevE.69.061603

16. A.M. Walsh, R.D. Coalson, J. Chem. Phys. 100(2), 1559 (1994)

17. N. Ben-Tal, R.D. Coalson, J. Chem. Phys. 101(6), 5148 (1994). DOI DOI:10.1063/1.467371

18. H. Kleinert, Path Integrals in Quatum Mechanics, Statistics, and Polymer Physics, 2nd edn. (World Scientific, Singapore, 1995)

19. Y. Levin, Reports on Progress in Physics 65, 1577 (2002). DOI 10.1088/0034-4885/65/11/201

20. R.A. Curtis, L. Lue, J. Chem. Phys. 123, 174702 (2005). DOI 10.1063/1.2102890

21. R.A. Curtis, L. Lue, Curr Opin. Colloid Interface Sci. 20(1), 19 (2015). DOI 10.1016/j.cocis.2014.12.001

22. S. Buyukdagli, M. Manghi, J. Palmeri, Phys. Rev. E 81(4), 041601 (2010)

23. S. Buyukdagli, C.V. Achim, T. Ala-Nissila, J. Chem. Phys. 137(10), 104902 (2012)

24. M.M. Hatlo, A. Karatrantos, L. Lue, Phys. Rev. E 80(6), 061107 (2009). DOI 10.1103/PhysRevE.80.061107

25. M.M. Hatlo, L. Lue, Soft Matter 5, 125 (2009). DOI 10.1039/b815578j

26. M.M. Hatlo, L. Lue, EPL (Europhysics Letters) 89(2), 25002 (2010). DOI 10.1209/0295$5075 / 89 / 25002$ 